\title{
Soft vortex matter in a type-I/type-II superconducting bilayer
}

\author{
L. Komendová, ${ }^{*}$ M. V. Milošević, and F. M. Peeters \\ Departement Fysica, Universiteit Antwerpen, Groenenborgerlaan 171, B-2020 Antwerpen, Belgium \\ (Received 22 January 2013; revised manuscript received 23 August 2013; published 23 September 2013)
}

\begin{abstract}
Magnetic flux patterns are known to strongly differ in the intermediate state of type-I and type-II superconductors. Using a type-I/type-II bilayer we demonstrate hybridization of these flux phases into a plethora of unique new ones. Owing to a complicated multibody interaction between individual fluxoids, many different intriguing patterns are possible under applied magnetic field, such as few-vortex clusters, vortex chains, mazes, or labyrinthal structures resembling the phenomena readily encountered in soft-matter physics. However, in our system the patterns are tunable by sample parameters, magnetic field, current, and temperature, which reveals transitions from short-range clustering to long-range ordered phases such as parallel chains, gels, glasses, and crystalline vortex lattices, or phases where lamellar type-I flux domains in one layer serve as a bedding potential for type-II vortices in the other, configurations clearly beyond the soft-matter analogy.
\end{abstract}

DOI: 10.1103/PhysRevB.88.094515

PACS number(s): 74.78.Fk, 64.60.Cn, 74.25.Uv, 82.70.Gg

\section{INTRODUCTION}

Soft-matter physics deals with systems as different as colloids, polymers, gels, glasses, liquid crystals, and others, where one common feature is their self-organization into very rich mesoscopic phases. ${ }^{1}$ To model this behavior, one often uses a pairwise interparticle interaction possessing several length scales and/or mixture of attraction and repulsion. ${ }^{2-9}$ Such interaction potential, as a function of the particle density, indeed leads to the formation of clusters, particle chains, labyrinthal gel-like structures, and (almost) regular lattices. This in turn questions the known analogy between charged colloids and vortices in superconductors, since the latter typically repel and form a triangular (Abrikosov) lattice. On the other hand, type-I superconductors are known to exhibit lamellar and labyrinthal flux patterns, which lose distinction of individual vortices but resemble the soft-matter structures in their macroscopic shape. ${ }^{10}$

With this in mind, we here investigate magnetic flux patterns in a coupled bilayer of two superconducting films-one type I and one type II- under perpendicular magnetic field (see Fig. 1), in an attempt to reveal unique vortex phases. In addition to the crystalline vortex lattice, one now envisages vortex flocculation, gelation, and glassy phases, some similar to vortex matter encountered in high-temperature, ${ }^{11}$ multiband, ${ }^{12}$ and other unconventional superconducting ${ }^{13}$ and superfluid systems. ${ }^{14}$ The film geometry is chosen for an easy realization in experiment, but also in order to have asymptotic long-range $1 / r$ repulsion between vortices, ${ }^{15}$ similar to the electrostatic Coulomb interaction in charged colloids. We will show that the complexity of the obtained patterns stems from the changes in the short- and midrange interaction between vortices, whose relative strength depends on the parameters of the layers, especially their coherence length $\xi$ and penetration depth $\lambda$, but also their thicknesses, electronic coupling between them, and chosen temperature with respect to their individual critical temperatures.

The paper is organized as follows. In Sec. II, we present the theoretical formalism. Section III summons and classifies the observed magnetic flux patterns, which are further characterized using radial distribution functions in Sec. IV. Further we discuss the influence of temperature in Sec. V, where we also show the behavior of the heat capacity and its changes affiliated with different flux phases. Our results are summarized in Sec. VI.

\section{THEORETICAL FORMALISM}

Most of earlier works on vortex structures and their dynamics employed molecular dynamics with pairwise vortex interactions. This is truly valid only for vortices in extreme type-II superconductors, where vortex cores are pointlike small. However, overlapping vortex cores do not interact pairwise, and the interaction potentials are highly nontrivial. ${ }^{16}$ This turns out to be even more complex for our bilayer system, where vortices are extended objects with different size of the core in two layers. We therefore opt for full numerical simulation within the Ginzburg-Landau (GL) theory, supplemented by the Lawrence-Doniach (LD) coupling between the layers. ${ }^{17-21}$ The appropriate free energy functional then consists of the individual contributions from each layer, the LD coupling term, and the energy of the magnetic field in and around the sample:

$$
\begin{aligned}
\mathcal{F}= & \sum_{j=1,2} d_{j} \int\left[\alpha_{j}\left|\Psi_{j}\right|^{2}+\frac{1}{2} \beta_{j}\left|\Psi_{j}\right|^{4}\right. \\
& \left.+\frac{1}{2 m_{j}}\left|\left(\frac{\hbar}{i} \nabla-\frac{2 e}{c} \mathbf{A}\right) \Psi_{j}\right|^{2}\right] d S \\
& +s \int \eta\left|p \Psi_{2}-\Psi_{1}\right|^{2} d S+\int \frac{(\mathbf{h}-\mathbf{H})^{2}}{8 \pi} d V .
\end{aligned}
$$

Here Cooper-pair condensates in the two layers are indexed by $j=1,2$ and described by the order parameters $\Psi_{j}(\mathbf{r})$, assumed to be uniform over the layer thickness $d_{j}$. $\mathbf{H}$ is the applied magnetic field and $\mathbf{h}=\operatorname{curl} \mathbf{A}$ is the field including the magnetic response of the layers. The coefficients $\alpha_{j}(T)=-\alpha_{j 0}\left(1-T / T_{c j}\right)$ are temperature dependent (where $T_{c j}$ are the critical temperatures of the individual layers) and connected with the nominal coherence lengths of the layers $\xi_{j}(T)=\hbar /\left|2 m_{j} \alpha_{j}(T)\right|^{1 / 2}=\xi_{j 0} / \sqrt{1-T / T_{c j}}$, where $m_{j}$ denotes Cooper-pair masses in the layers. The LD coupling coefficient is $\eta=\hbar^{2} /\left(2 m_{\perp} s^{2}\right)$, where $m_{\perp}$ is the effective Cooper pair mass for tunneling between the layers and $s$ is 


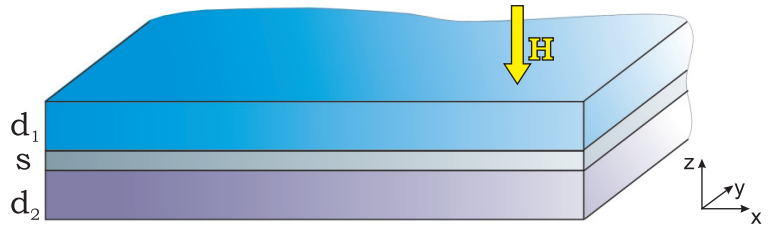

FIG. 1. (Color online) The oblique view of the considered bilayer sample. The two superconducting layers are separated by an ultrathin oxide/insulating layer. The magnetic field is applied in the direction perpendicular to the layers (along the $z$ axis).

the vertical distance between the layers (see Fig. 1). The phase factor $p=\exp \left(-i \frac{2 e}{\hbar c} \int_{0}^{s} A_{z} d z\right)$ ensures the gauge invariance.

In what follows, we work in the London gauge $\boldsymbol{\nabla} \cdot \mathbf{A}=0$; therefore the Maxwell equation is just

$$
-\Delta \mathbf{A}=\frac{4 \pi}{c} \mathbf{j} .
$$

Further we make approximation $A_{z}=0$, so that the phase factor $p$ in the LD coupling term is unity (a similar model was also employed in Ref. 22). Since $A_{z}=0$, Eq. (2) implies also $j_{z}=0$. On the other hand, in the full model the current between the layers is

$$
\begin{aligned}
j_{z} & =\frac{i e \hbar}{m_{\perp} s}\left[\psi_{1}^{*} p \psi_{2}-p^{*} \psi_{2}^{*} \psi_{1}\right] \\
& =\frac{2 e \hbar}{m_{\perp} s}\left|\psi_{1}\right|\left|\psi_{2}\right| \sin \left(\varphi_{1}-\varphi_{2}\right),
\end{aligned}
$$

where we used $p=1$ and $\psi_{j}=\left|\psi_{j}\right| e^{i \varphi_{j}}$. The current between the layers $j_{z}$ can be neglected if it is much smaller than the currents within the superconducting layers

$$
\mathbf{j}_{j}=-\frac{i e \hbar}{m_{j}}\left(\Psi_{j}^{*} \nabla \Psi_{j}-\Psi_{j} \nabla \Psi_{j}^{*}\right)-\frac{4 e^{2}}{m_{j} c}\left|\Psi_{j}\right|^{2} \mathbf{A},
$$

which leads to the condition

$$
\frac{m_{j}}{m_{\perp}} \frac{\xi_{j}(T)}{s} \sin \left(\phi_{1}-\phi_{2}\right) \ll 1,
$$

if we assume that the amplitudes of the wave functions in two layers are not too different from each other. Assuming $A_{z}=0$ is thus well justified for $m_{\perp} \gg m_{j}$ or very distant layers $s \gg \xi_{j}(T)$, i.e., in the case of very weakly coupled layers, but also in the opposite case $m_{\perp} \approx m_{j}$ where the phases of the two order parameters are almost the same, so that the term $\sin \left(\phi_{1}-\phi_{2}\right)$ vanishes. Therefore, for the parameters of the sample chosen in the present work $j_{z}$ is always much smaller than the current within the superconducting layers and one can safely neglect it.

The variational minimization of the functional (1) (with $p=1$ ) with respect to $\Psi_{j}^{*}$ leads to the GLLD equations

$$
\frac{1}{2 m_{1}}\left(\frac{\hbar}{i} \nabla-\frac{2 e}{c} \mathbf{A}\right)^{2} \Psi_{1}+\tilde{\alpha}_{1} \Psi_{1}+\beta_{1}\left|\Psi_{1}\right|^{2} \Psi_{1}-\eta \frac{s}{d_{1}} \Psi_{2}=0,
$$

$\frac{1}{2 m_{2}}\left(\frac{\hbar}{i} \nabla-\frac{2 e}{c} \mathbf{A}\right)^{2} \Psi_{2}+\tilde{\alpha}_{2} \Psi_{2}+\beta_{2}\left|\Psi_{2}\right|^{2} \Psi_{2}-\eta \frac{s}{d_{2}} \Psi_{1}=0$, where $\tilde{\alpha}_{j}=\alpha_{j}+\eta s / d_{j}$. We solve this system of equations numerically, self-consistently with the equations for the supercurrent density per unit volume in each layer given by Eq. (4). Note that the supercurrents only flow inside the respective layers and therefore are spatially separated. Substituting Eq. (4) into Eq. (2) with $\mathbf{j}=\mathbf{j}_{1}+\mathbf{j}_{2}$ provides the total $3 \mathrm{D}$ vector potential $\mathbf{A}$, which we calculated in the middle of each layer and used iteratively in respective Eqs. (6a) and (6b) to compute $\Psi_{1}$ and $\Psi_{2}$ (for details of the procedure, see Ref. 23).

We performed the numerical simulations on a rectangular region, with aspect ratio $2: \sqrt{3}$ and periodic boundary conditions. We chose the parameter values close to a clean $\mathrm{Nb}$ film as the type-II layer (with GL parameter $\kappa_{1}=1.03, T_{c 1}=9.2$ $\mathrm{K})$ and $\mathrm{Sn}$ as the type-I layer $\left(\kappa_{2}=0.15, T_{c 2}=3.7 \mathrm{~K}\right)$. The lowest considered temperature was $1 \mathrm{~K}$, which was necessary to be deep in the superconducting state of the type-I layer. Zero-temperature coherence length of the type-II layer $\left(\xi_{10}\right)$ was then taken as unit of distance in all calculations, while $\xi_{20}$ was swept between 2 and $10 \xi_{10}$. Since this variation had only minor qualitative influence on the observed vortex structures, we fixed the parameter $\zeta=\left(\xi_{10} / \xi_{20}\right)^{2}$ to 0.2 , for computational convenience. Order parameters were scaled to $\Psi_{j 0}=\sqrt{-\alpha_{j}(0) / \beta_{j}}$, and magnetic field to $H_{0}=\Phi_{0} /\left(2 \pi \xi_{10}^{2}\right)$, where $\Phi_{0}$ is the flux quantum.

\section{PHASE DIAGRAM AS A FUNCTION OF APPLIED MAGNETIC FIELD AND COUPLING BETWEEN THE LAYERS}

In Fig. 2, we show the key result of our paper, the vortex phase diagram of the described bilayer at $T=1 \mathrm{~K}$ as a function of the applied magnetic field and the effective mass

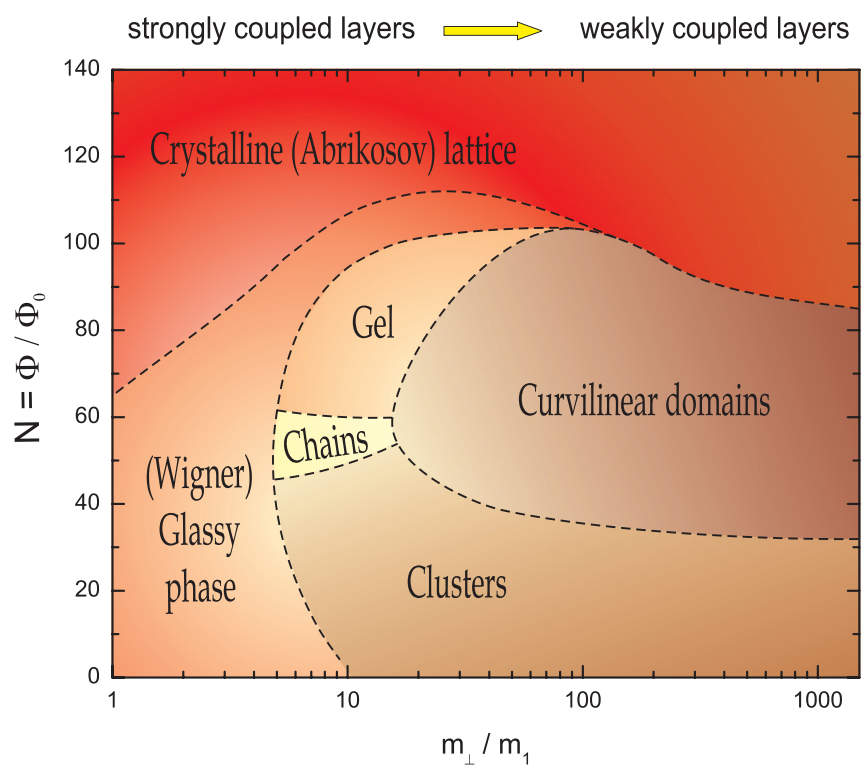

FIG. 2. (Color online) The equilibrium phase diagram of a Nb/Sn bilayer calculated at $T=1 \mathrm{~K}$, for both layers $5 \xi_{10}$ thick and spacer layer of $0.05 \xi_{10}$ in between, as a function of the applied field (expressed through the number of flux quanta $N$ in the simulation region $55 \times 47.6 \xi_{10}^{2}$ ) and effective mass $m_{\perp}$ of the Cooper pairs in the spacer layer. When other parameters are fixed, the electronic coupling between the superconducting layers is inversely proportional to $m_{\perp}$. 


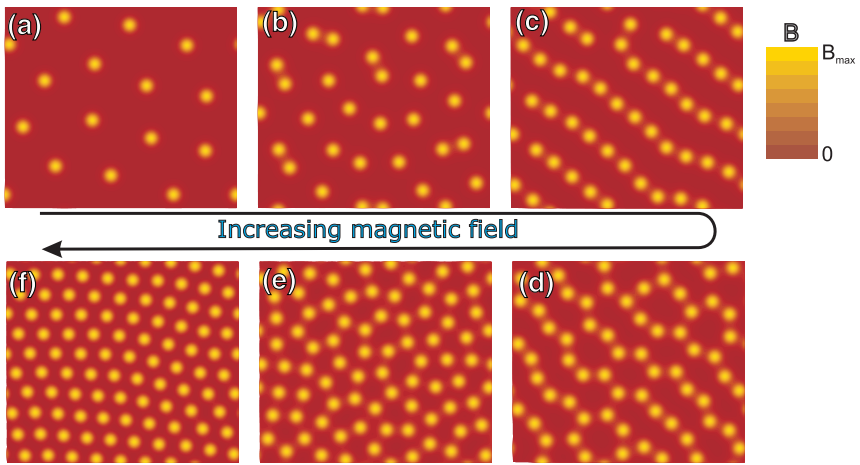

FIG. 3. (Color online) Very strongly coupled layers. Vortex structure is shown via normalized magnetic field profile in the type-II layer (identical vortex structure is found in the type-I layer), for states found in Fig. 2 for $m_{\perp}=5 m_{1}$. In panels (a)-(f) there are 16, 32, 48, 64,80 , and 96 vortices in the simulation region, respectively.

in the spacer layer, which controls the strength of the coupling between the superconducting layers. The field is expressed through the number of vortices in the simulation region. We revealed a series of nonuniform vortex phases, with phase transitions between them indicated by dashed curves in the diagram. In what follows, we discuss these transitions by showing exemplary vortex configurations.

Figure 3 shows obtained vortex configurations for very strongly coupled layers $\left(m_{\perp}=5 m_{1}\right)$. At low fields we observe a quasihomogeneous distribution of vortices, indicative of long-range repulsion between them, reminiscent of Wigner glass in soft-matter physics (see, e.g., Ref. 8). However, with increasing field, after reaching some threshold vortex density, vortex dimers, short individual chains, and then long-ordered parallel chains are formed, in complete analogy to what was seen in Ref. 2 for particle systems with purely repulsive interactions but two governing length scales [i.e., a potential with very strong repulsion at short range ("hard core"), flattening at midrange ("soft core"), and then abruptly weaker repulsion at long range]. The half-distance between the parallel vortex chains in Fig. 3(c) then gives an estimate of the "soft core." As the vortex density is further increased, the chains interconnect into a glassy disordered structure, and finally form the crystalline (Abrikosov) lattice. The intervortex distance at the latter glass-solid transition then provides an estimate of the "hard core" of the repulsive vortex interaction. Notably, similar states as here have also been recently found in Ref. 5 for a phenomenologically introduced potential with a repulsive core surrounded by an attractive well and finally a long-range repulsion.

In Fig. 4, we reduced the coupling between the layers with a factor 2 as compared to Fig. 3 (i.e., we increased $m_{\perp}$ to $10 m_{1}$ ). The found vortex configurations are very different, starting at low fields from small clusters of 2-4 vortices, combined with short chains. With further increase of the magnetic field the chains establish long-range order, then curve, recombine, and finally interconnect into a low-density network filling the entire simulation region. Such a network, that spans the volume of the medium while at low particle density, is typical for gels. This gel-like structure is retained in Fig. 4 until the newly added vortices fill all the voids in the gel and form a disordered

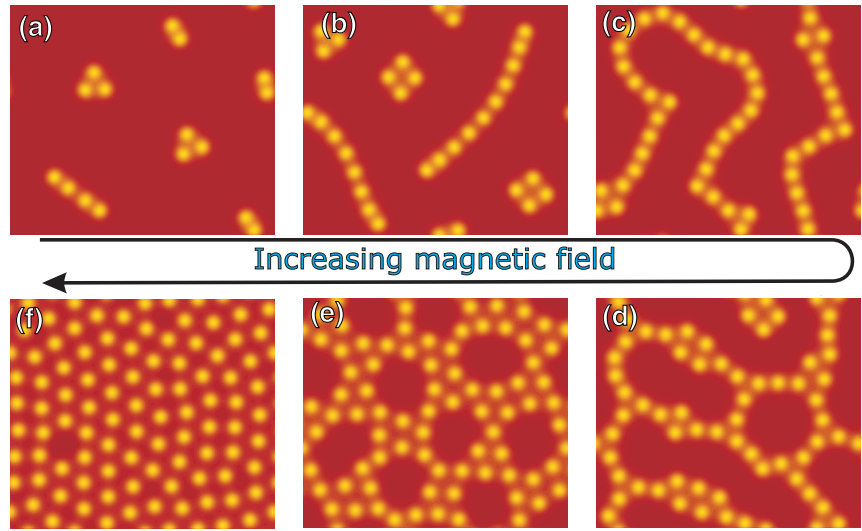

FIG. 4. (Color online) Strongly coupled layers. Same as Fig. 3, but for twice weaker coupling between the layers, i.e., $m_{\perp}=10 m_{1}$. In panels (a)-(e) there are 16, 32, 48, 64, 80, and 96 vortices in the simulation region, respectively.

(glassy) lattice, similarly to the case of Fig. 3, followed by crystallization at high vortex density.

In Fig. 5, the states are shown for $m_{\perp}=15 m_{1}$, which is still a relatively strong coupling. For low fields tiny clusters are formed, which then give way to prolonged chains, gel states, and finally a (quasi)crystalline lattice for 128 vortices in the simulation box (not shown). One can see that $m_{\perp}=15 m_{1}$ lies on the crossover of stability regions of spatial structures with lateral extent one and two vortices, i.e., at the transition from chains to curvilinear domains in Fig. 2. In Fig. 6, the electronic coupling between superconducting layers is further decreased $\left(m_{\perp}=20 m_{1}\right)$, and the qualitative trend of Fig. 5 is maintained. With increasing field, a transition from a solution of clusters to short-range mazes and then long-range gel is found, followed by crystallization of the vortex lattice. However, the superconducting type-I behavior of the type-I layer becomes more apparent, as vortices become less distinct from each other, and occupy increasingly wider domains. This is more clearly demonstrated in Fig. 7, where we gradually

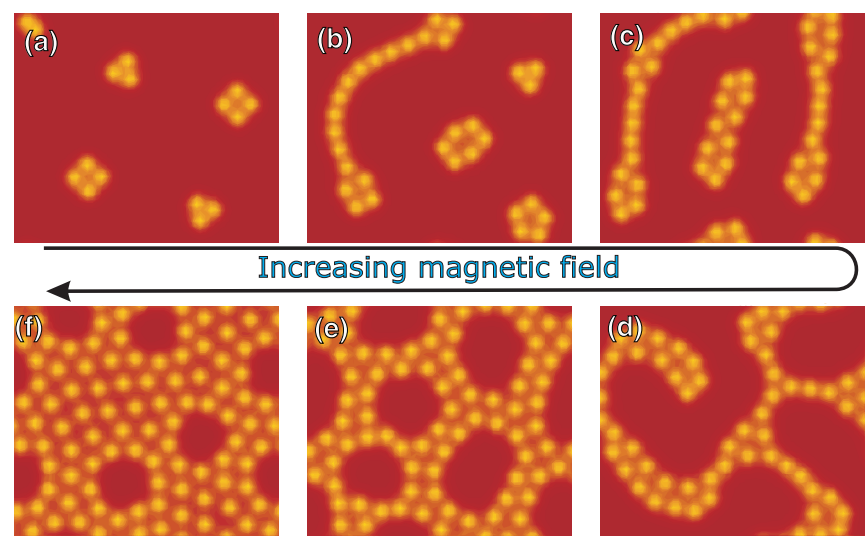

FIG. 5. (Color online) Evolution of the states with applied magnetic flux, at coupling $m_{\perp} / m_{1}=15$ (cf. Fig. 2). The spatial profile of the magnetic field is shown for (a)-(f) $16,32,48,56,80$, and 96 vortices, respectively. In this sequence of images, we sample the found phases in the busiest region of the phase diagram shown in Fig. 2. Transitions between clusters and chains, to mazes and gel are shown. 


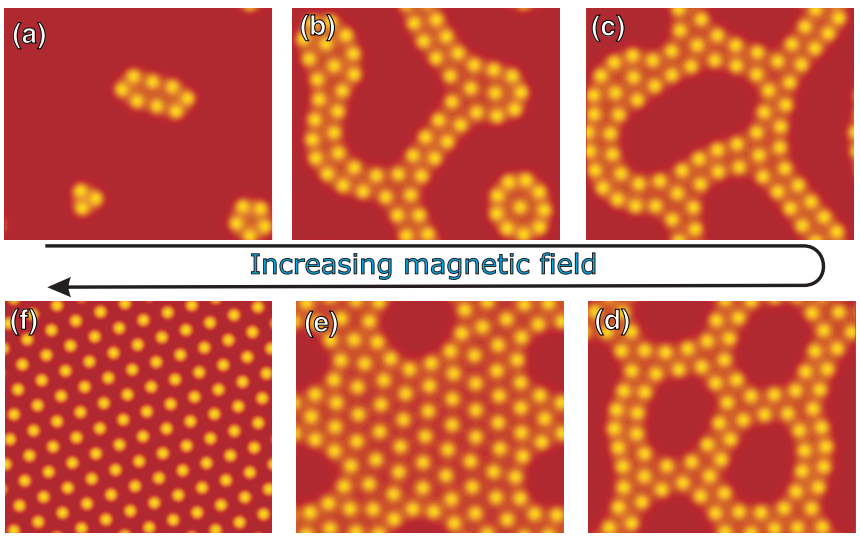

FIG. 6. (Color online) Intermediately strongly coupled layers. Same as Figs. 3-5 but for further weakened coupling between layers, i.e., for $m_{\perp}=20 m_{1}$. In panels (a)-(f) there are 16, 64, 72, 80, 96, and 112 vortices in the simulation region, respectively.

increased the coupling between the layers for a fixed number of vortices in the simulation. Notice that the lateral extent of the vortex stripes varies from just one vortex for strong coupling, to four vortices for weak coupling. This behavior is reminiscent of the colloidal structures studied in Ref. 3, where particles interacted via Coulomb-like repulsion combined with a midrange attraction, and where the widening of the stripe phases was directly linked to the increasing strength and range of the attractive part of the interaction. It is interesting to note that a very similar state to Fig. 7(f) was found in Ref. 24 in the presence of a dominant three-body interaction. This suggests that although most of our above results can be understood using pairwise interactions, multibody interactions may nevertheless play a significant role.

For even smaller values of interlayer coupling one can no longer rely on a two-body vortex-vortex interaction, since the type-I layer exhibits laminar domains, and the notion of individual vortices is completely lost. In Fig. 8 we depict the changes in vortex patterns in a broad range of $m_{\perp}$ values, where formation of type-I domains is visible for $m_{\perp}>20 m_{1}$. Those domains act as potential wells for vortices in the

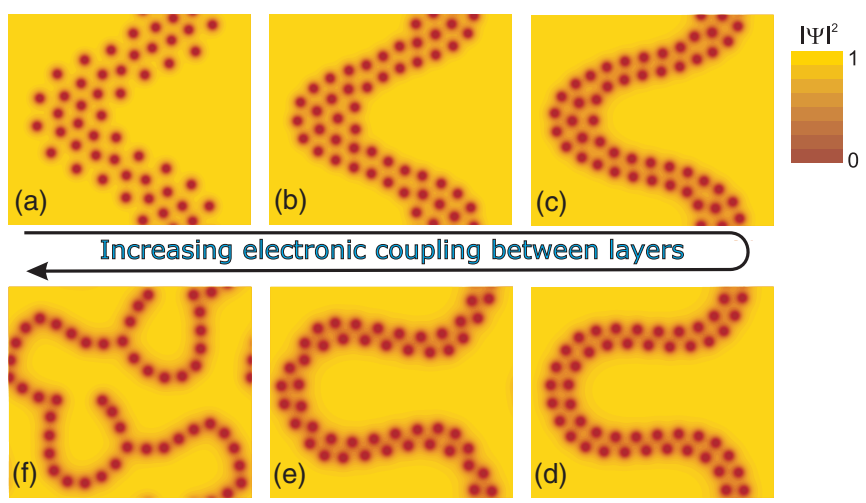

FIG. 7. (Color online) The evolution of the vortex structure with decreasing $m_{\perp}$, i.e., with increasing strength of the electronic coupling between the layers, for 48 vortices in the simulation region. In panels (a)-(f), the normalized Cooper-pair density in the type-II layer is shown for $m_{\perp} / m_{1}=160,120,60,30,20$, and 10 , respectively.
Type-II layer

(a)

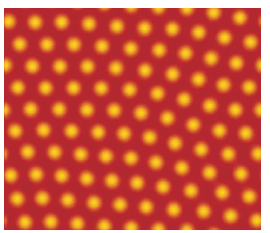

(b)

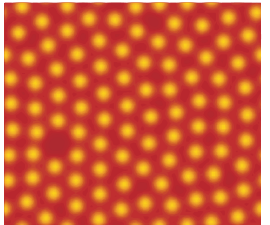

(c)

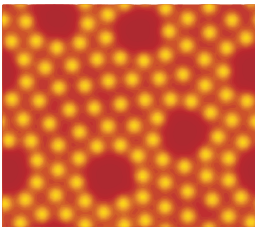

(d)

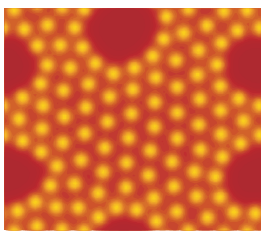

(e)

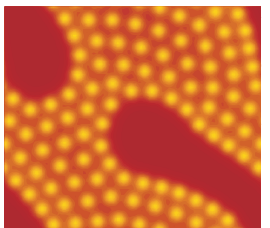

(f)

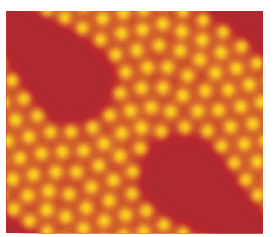

(g)

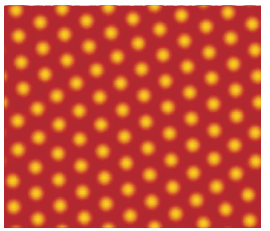

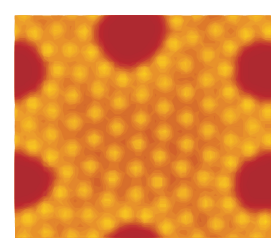
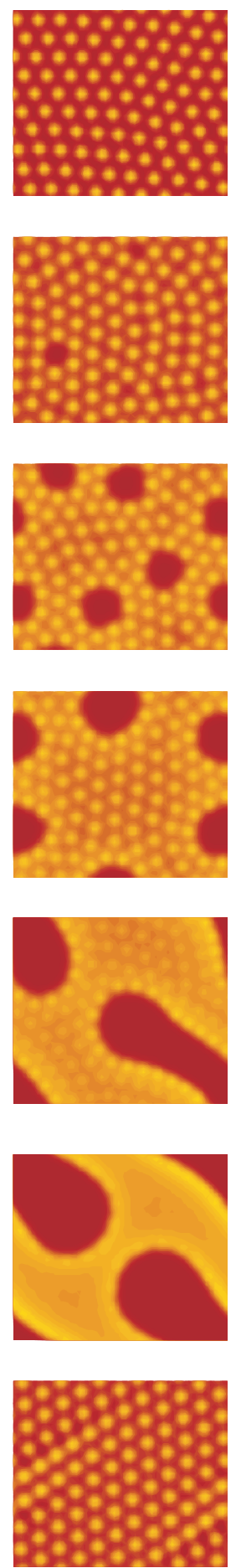

FIG. 8. (Color online) Evolution of the vortex states with decreasing coupling, at fixed magnetic flux (96 vortices; cf. Fig. 2). The spatial profile of the magnetic field is shown. From top to bottom $m_{\perp} / m_{1}=$ (a) 5, (b) 10 , (c) 15 , (d) 20, (e) 40, (f) 200, and (g) 300 . Left panel shows the type-II layer and the right one the type-I layer. In this sequence of images, we show the found phases going left to right in the phase diagram shown in Fig. 2. Transitions between Abrikosov lattice, gels, and type-I domains decorated by type-II vortices are found. 


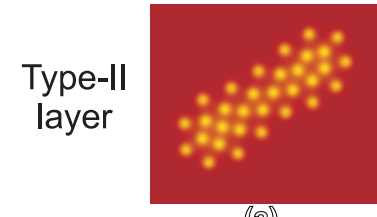

(可)
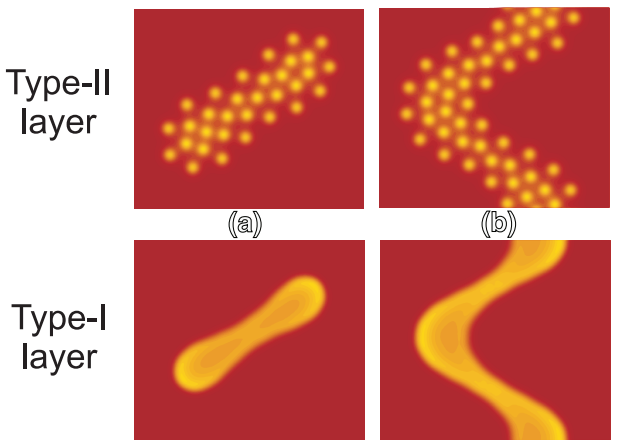

(b)
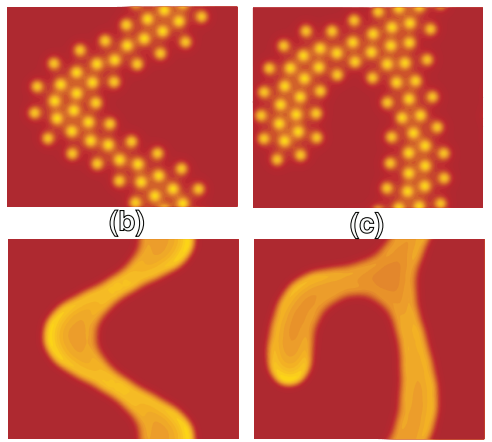

(ब)

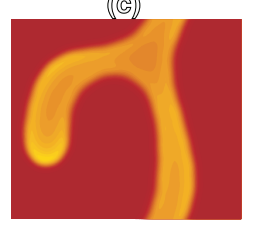

FIG. 9. (Color online) Weakly coupled layers. Flux patterns are shown in the type-II layer (top row) and the type-I layer (bottom row), for states found in Fig. 2 for $m_{\perp}=1000 m_{1}$. In panels (a)-(c) there are 32,48 , and 64 flux quanta in the simulation region, respectively.

type-II layer, as exemplified in Fig. 9, which shows states for $m_{\perp}=1000 m_{1}$. Because of this, the structural phase transitions become different from the $m_{\perp}<20 m_{1}$ cases. This is clearly seen in the change of curvature of the sol-gel and the gel-glass transition lines in Fig. 2. With decreasing coupling, due to the easier formation of type-I domains, vortices in the type-II layer connect into mazes at lower densities. On the other hand, they also crystallize at lower densities than for strong coupling, which is due to the practically destroyed superconductivity in the type-I layer at such a large magnetic field. The formation of domains in the type-I layer as a trapping potential for vortices is interesting because of their sensitivity to applied in-plane magnetic field, ${ }^{25}$ or current. ${ }^{26}$ Therefore one can easily manipulate externally the domain structure in the type-I layer (e.g., straighten/relax the domains), and thereby dynamically change and restore the vortex patterns in the type-II layer, similarly to the controllability achieved in the superconductor-ferromagnet bilayers. ${ }^{27}$

\section{RADIAL DISTRIBUTION FUNCTIONS OF OBSERVED CONFIGURATIONS}

In this section we present the radial distribution functions $g(r)$ of different vortex phases observed in the superconducting type-I/type-II bilayer, which can in some cases serve to distinguish particular vortex configurations in, e.g., SANS measurements.

The radial distribution function $g(r)$ characterizes the particle distribution. It gives clear signatures of the crystalline order and it can be also experimentally determined, e.g., by neutron scattering. It is defined as

$$
g(r)=\frac{1}{N} \frac{\rho(r)}{\rho},
$$

where $\rho(r)$ is the density of particles at some distance $r$ from the origin, while $\rho=N / A$, with $N$ the total number of particles and $A$ the total area, is the average density. The density $\rho(r)$ is in practice computed from the histogram of all distances between the pairs of particles, where the number of particles $N_{i}$ in each bin $\left[r_{i}-d r / 2 ; r_{i}+d r / 2\right]$ is divided by the area corresponding to that bin $A_{i}=$ $\pi\left[\left(r_{i}+d r / 2\right)^{2}-\left(r_{i}-d r / 2\right)^{2}\right]$. Note that each pair counts for two particles. Taking this into account, we calculated $g(r)$
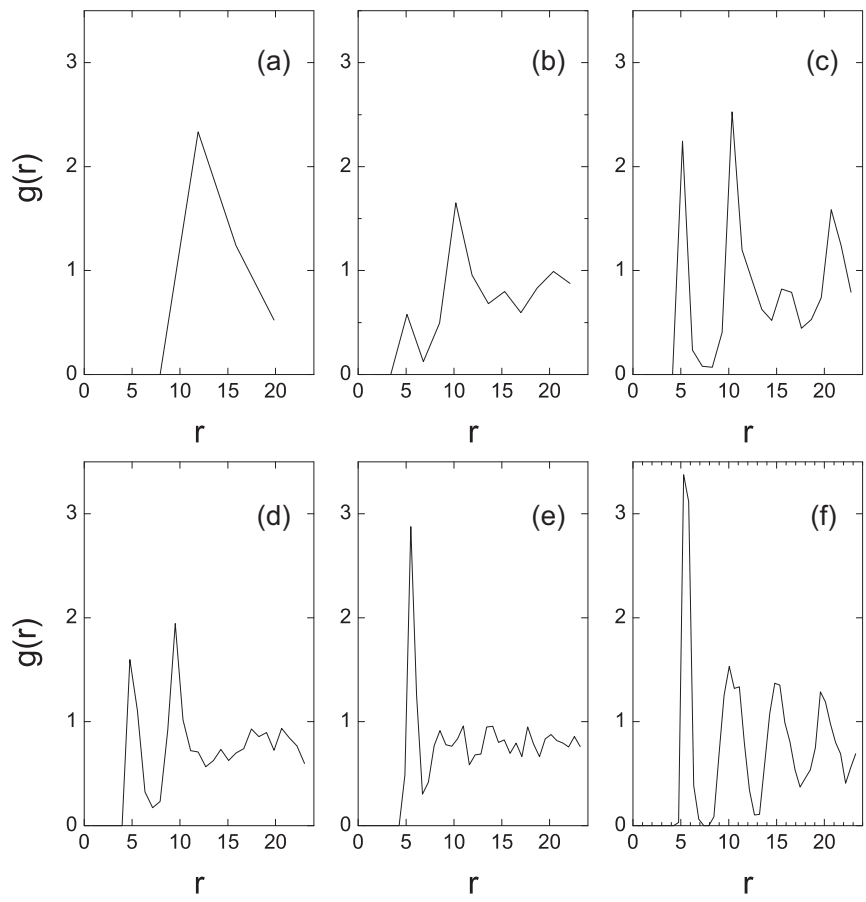

FIG. 10. The radial distribution function $g(r)$ for $m_{\perp}=5 m_{1}$. Panels (a)-(f) correspond to states with 16, 32, 48, 64, 80, and 96 vortices in the simulation region, respectively.

using the formula

$$
g(r)=\frac{2 N_{i}}{N \rho \pi\left[\left(r_{i}+d r / 2\right)^{2}-\left(r_{i}-d r / 2\right)^{2}\right]} .
$$
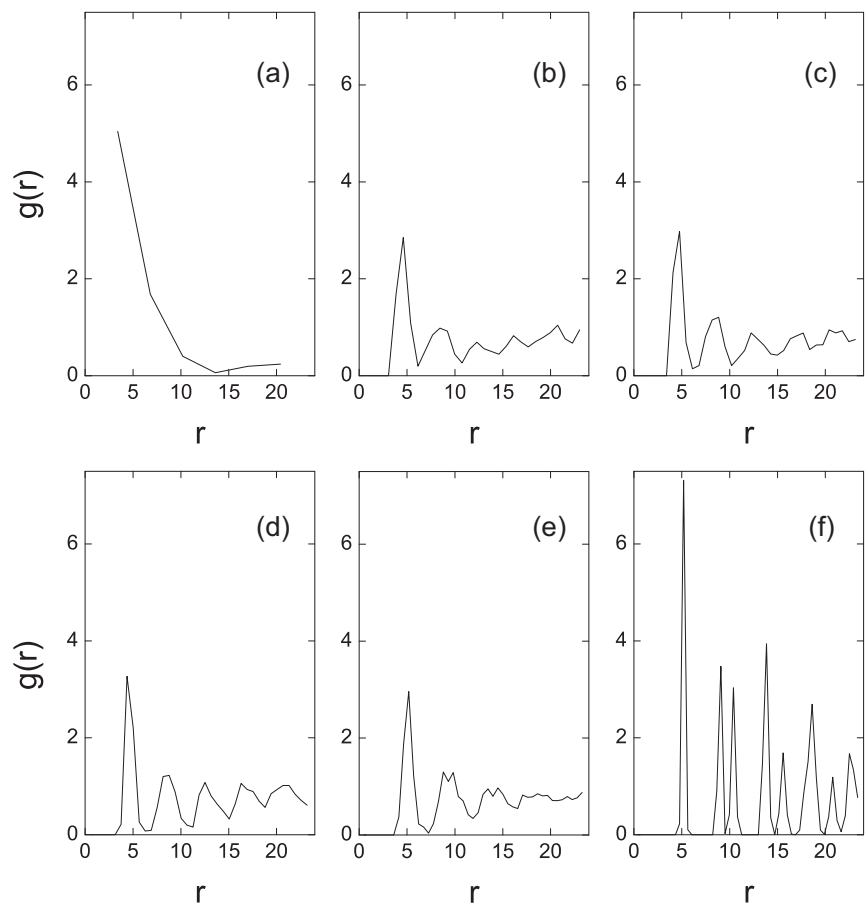

FIG. 11. The radial distribution function $g(r)$ for $m_{\perp}=20 m_{1}$. Panels (a)-(f) correspond to states with 16, 64, 72, 80, 96, and 112 vortices in the simulation region, respectively. 


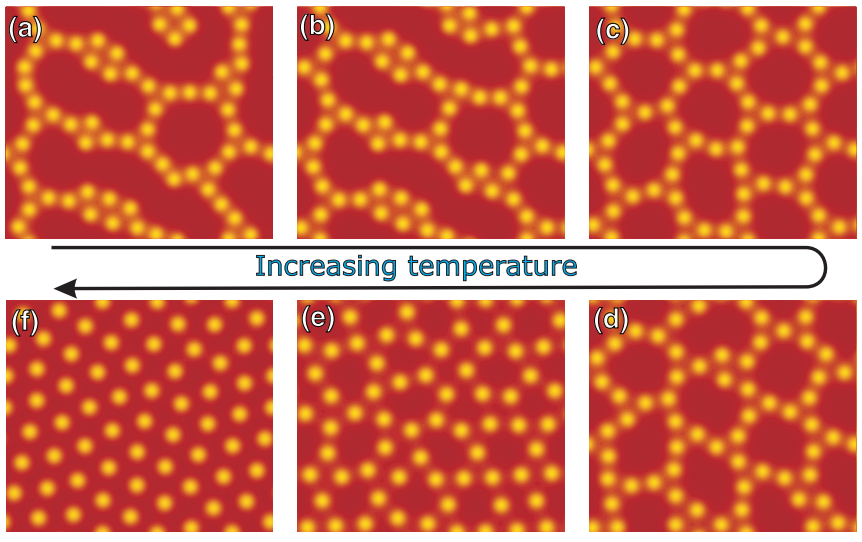

FIG. 12. (Color online) The evolution of the gel-like phase of Fig. 4(d) with increasing temperature. The vortex structure in the type-II layer is shown for (a) $T=1 \mathrm{~K}$ (original state) and for the field-heated states at (b) $T=1.1 \mathrm{~K}$, (c) $1.25 \mathrm{~K}$, (d) $1.45 \mathrm{~K}$, (e) 1.85 $\mathrm{K}$, and (f) $2.2 \mathrm{~K}$.

In Figs. 10 and 11 we show the radial distribution function for the states displayed in Figs. 3 and 6, respectively. In each case we use the total number of bins equal to half of the total number of vortices. The distance between any two vortices is determined as the distance to the nearest periodic image. In order not to account the interaction of vortex with its own periodic image, we only show $g(r)$ for $r$ up to $23 \xi_{10}$, i.e., approximately half of the shorter side of our simulation region.

The $g(r)$ functions for smaller densities, i.e., for 16 and 32 vortices in the simulation region, are too coarse to be useful; however in the radial distribution functions for our simulations for larger applied magnetic fields we can see important signatures of the order. For example in Fig. 11 in panels (e) and (f) for 96 respectively 112 vortices, one can see that the second peak splits into two, which is a well-known effect due to the formation of the regular triangular (Abrikosov) lattice (in the first case with holes of the Meissner state still present). One can also see in Fig. 10 that the chain phase for $m_{\perp} / m_{1}=5$ and intermediate densities (48 and 64 vortices) can be distinguished by having the second peak of $g(r)$ higher than the first one. Similar signature was also observed in Ref. 2, but there it was found for the structure factor $S(k)$, i.e., in the $k$ space. This signature disappears when a gel state is formed.

\section{PHASE TRANSITIONS BETWEEN DIFFERENT STRUCTURES WITH TEMPERATURE}

Another degree of controllability of the flux patterns in our system is provided by temperature, due to the different critical temperatures $T_{c j}$ of the layers. For the considered parameters, elevated temperature will swiftly deplete superconductivity in the type-I layer, and will also interconnect the flux patterns in that layer due to the increasing coherence length. Both these features will influence the observed vortex configurations in the type-II layer. Hence, one is able to control and monitor the phase transitions of soft vortex matter in our bilayer system simply by changing temperature. We illustrate this in Fig. 12, where we start from a rather disordered gel-like state from Fig. 4(d) and gradually increase temperature. After

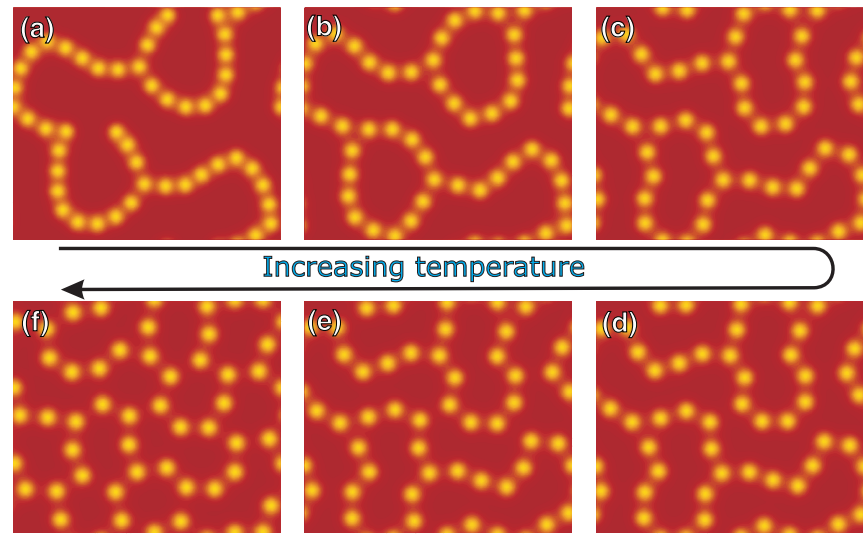

FIG. 13. (Color online) Evolution of the state shown in Fig. 7(f), with increasing temperature. The spatial profile of the magnetic field is shown. In this sequence of images, we show the transition from the chain phase to the gel phase by gradual increase of temperature. (a)-(f) $T=1 \mathrm{~K}, 1.5 \mathrm{~K}, 1.75 \mathrm{~K}, 1.85 \mathrm{~K}, 1.9 \mathrm{~K}$, and $2 \mathrm{~K}$.

a transition to a honeycomb (or fishing net) structure at $T=1.25 \mathrm{~K}$, a transition to a glassy phase was found at $1.8 \mathrm{~K}$, followed by crystallization into the Abrikosov lattice at $2.1 \mathrm{~K}$ (where the magnetic influence of the type-I layer became negligible). It is therefore interesting to note that contrary to most natural structures, including soft matter, the vortex configurations in our system become more ordered with increasing temperatures. Similarly, in Fig. 13 one can see how increasing the temperature transforms the chain state of Fig. 7(f) to the gel phase.

To gain insight into the nature of the phase transitions between different spatial arrangements of vortices, we show one particular example calculated for 16 vortices, thickness of the coupling layer $s=2 \xi_{10}$ and effective mass for tunneling of the Cooper pairs between the layers $m_{\perp}=40 m_{1}$. We found that for these parameters the stable phase at low temperature is small vortex clusters. These for intermediate temperatures coalesce into a single stripe which at higher temperature spreads over the entire simulation region and interconnects with adjacent stripes into the Abrikosov lattice. However, we found that there is considerable hysteretic behavior, since Fig. 14 shows the transition from several clusters to a stripe between $3.1 \mathrm{~K}$ and $3.15 \mathrm{~K}$ with increasing temperature, while on cooling (Fig. 15) the stripe is stable down to a much lower temperature of $0.85 \mathrm{~K}$. In order to characterize this phase transition, we calculate the free energy and heat capacity. The free energy is given by Eq. (1) which in dimensionless form reads

$$
\begin{aligned}
\frac{\mathcal{F}}{\mathcal{F}_{0}}= & d_{1} \int\left[-\chi_{1}\left|\Psi_{1}\right|^{2}+\frac{1}{2}\left|\Psi_{1}\right|^{4}+\left|(-i \nabla-\mathbf{A}) \Psi_{1}\right|^{2}\right] d S \\
& +C_{1} d_{2} \int\left[-\chi_{2}\left|\Psi_{2}\right|^{2}+\frac{1}{2}\left|\Psi_{2}\right|^{4}+\frac{1}{\zeta}\left|(-i \nabla-\mathbf{A}) \Psi_{2}\right|^{2}\right] d S \\
& +C_{2} \int\left|\Psi_{1}-C_{3} \Psi_{2}\right|^{2} d S+\kappa_{1}^{2} \int\left(\mathbf{A}-\mathbf{A}_{0}\right) \cdot \mathbf{j} d V,
\end{aligned}
$$

where $\mathcal{F}_{0}=\xi_{10}^{3} \alpha_{10}^{2} / \beta_{1}=\Phi_{0}^{2} /\left(32 \pi^{3} \kappa_{1}^{2} \xi_{10}\right)$ is our unit of en$\operatorname{ergy}\left(\mathcal{F}_{0} \approx 1.07 \times 10^{-18} \mathrm{~J}\right.$ for Niobium with $\left.\xi_{10}=38 \mathrm{~nm}\right)$, $\chi_{j}=1-T / T_{c j}, \mathbf{A}_{0}$ is the vector potential of the applied 

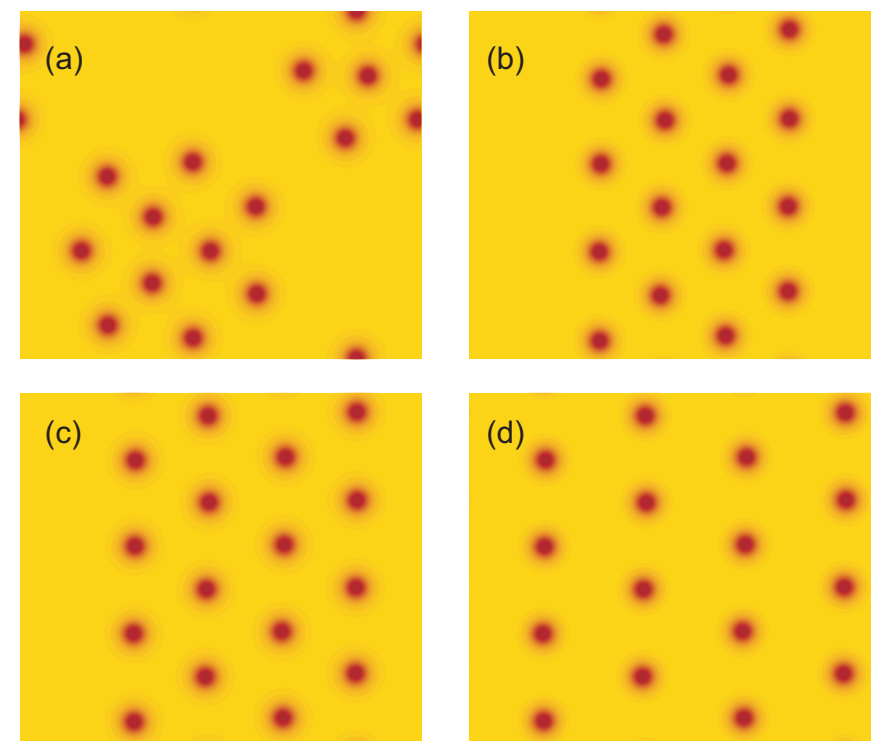

FIG. 14. (Color online) The transition from the cluster phase through stripe phase to the Abrikosov lattice on heating, shown as Cooper-pair density plots in the type-II layer at (a) $T=3.1 \mathrm{~K}$ (clusters), (b) $T=3.15 \mathrm{~K}$, (c) $T=3.25 \mathrm{~K}$ (stripe), and (d) $T=3.3 \mathrm{~K}$ (Abrikosov lattice).

field, $\mathbf{j}$ the supercurrent, and $C_{1}=\zeta^{2} \frac{\kappa_{1}^{2}}{\kappa_{2}^{2}}, C_{2}=\frac{m_{1}}{s m_{\perp}}$, and $C_{3}=\frac{\kappa_{1}}{\kappa_{2}} \sqrt{\zeta \frac{m_{2}}{m_{1}}}$.

In Fig. 16(a) we show the specific heat capacity vs temperature, computed as $c_{v}=-\frac{T}{V} \frac{\partial^{2} \mathcal{F}}{\partial T^{2}}$. We convert the specific heat capacity from the units of $\mathcal{F}_{0} /(V \mathrm{~K})$ (free energy per volume per kelvin) to its equivalent $\mathrm{SI}$ value which is in our case $6.2 \times 10^{-7} \mathrm{~J} \mathrm{~K}^{-1} \mathrm{~cm}^{-3}$ (using volume of the simulation region $V=31416 \xi_{10}^{3} \approx 1.72 \times 10^{-12} \mathrm{~cm}^{3}$ ). The most
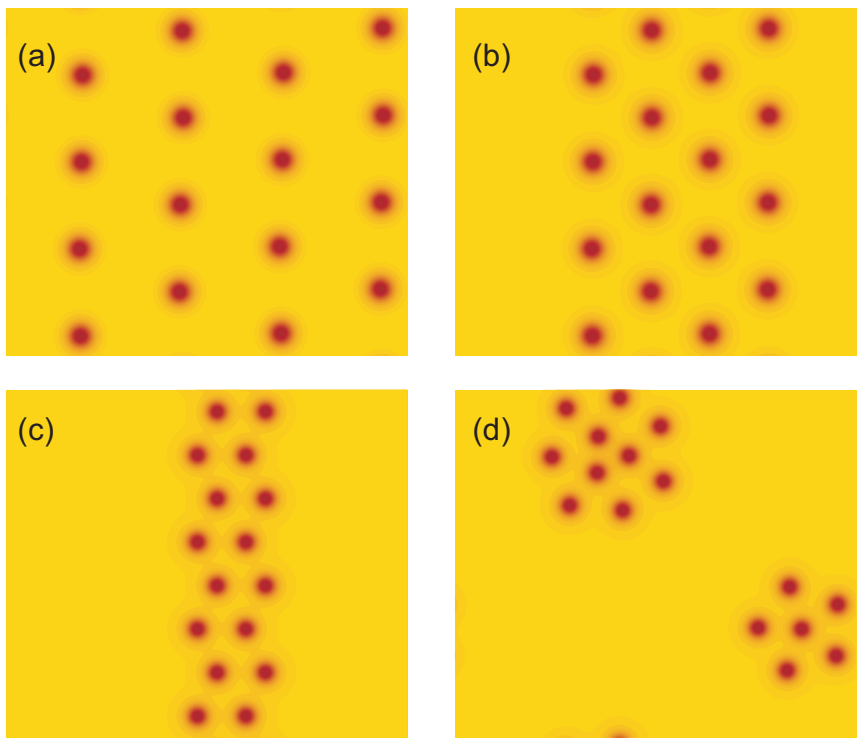

FIG. 15. (Color online) The transition from the Abrikosov lattice through stripe phase to the cluster phase on cooling, shown as Cooperpair density plots in the type-II layer at (a) $T=3.15 \mathrm{~K}$ (Abrikosov lattice), (b) $T=3.1 \mathrm{~K}$, (c) $T=0.85 \mathrm{~K}$ (stripe), and (d) $T=0.84 \mathrm{~K}$ (clusters).
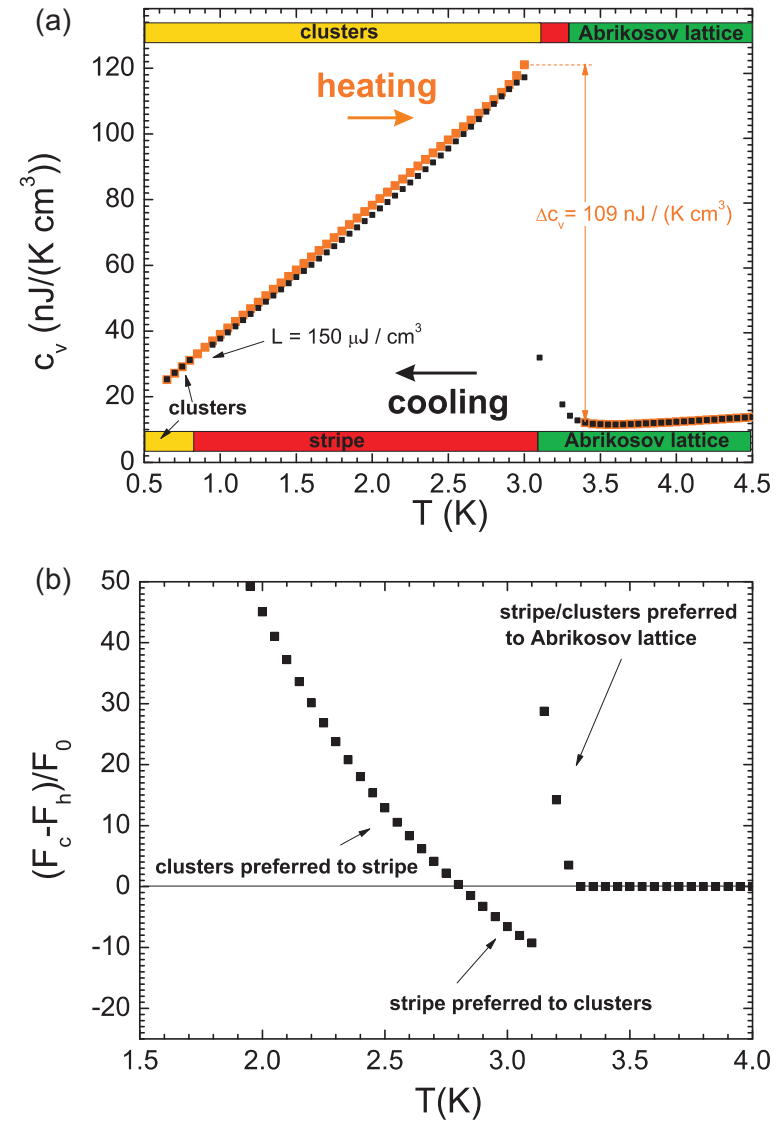

FIG. 16. (Color online) (a) The hysteretic behavior between the cluster phase, the stripe phase, and the Abrikosov lattice, shown via calculated heat capacity $c_{v}$ on heating and cooling. The top (bottom) banner labels the states found on heating (cooling). The main difference is that on heating the stripes are only found in a narrow temperature region close to $T=3.15 \mathrm{~K}$, while on cooling they are stable down to $0.85 \mathrm{~K}$. (b) The difference in the free energy $\mathcal{F}$ between the states found on cooling $\left(\mathcal{F}_{c}\right)$ and on heating $\left(\mathcal{F}_{h}\right)$.

pronounced feature in the specific heat capacity curve is a jump $\Delta c_{v}$ of approximately $109 \mathrm{~nJ} \mathrm{~K}^{-1} \mathrm{~cm}^{-3}$, where on heating the transition from small clusters to a single stripe is immediately followed by the rapid transition of the type-I layer through its own $T_{c}$ to only proximity-induced superconductivity. After that transition the vortices occupy the entire sample evenly, forming an Abrikosov lattice. Therefore we can associate the onset of the attraction between vortices directly with the onset of superconductivity in the type-I layer. This implies that for considered parameters the type-I layer must be below its own critical temperature in order to observe any unusual clustering of vortices.

On cooling the heat capacity shows a similar jump, but associated only with the transition from Abrikosov lattice to the stripe phase. The subsequent transition at $0.85 \mathrm{~K}$ corresponds to the rearrangement of the vortices from the stripe into clusters, it is of first order, and associated with latent heat $L \approx 150 \mu \mathrm{J} \mathrm{cm}^{-3}$. The described features in the heat capacity are ideal for observing the phase transitions of soft vortex matter by calorimetry, similarly to what is proposed for detection of flux phases inside the 3D samples and distinction of giant vortex to multivortex transitions in Ref. 31 . The exper- 
imental realization of the required high-precision calorimetry is feasible, and has already been reported in Ref. 32 .

In Fig. 16(b) we then show the difference in the free energies $\mathcal{F}_{c}-\mathcal{F}_{h}$ between the states found on cooling and heating, respectively. The sign of this quantity determines which of the possible states is energetically favorable and details the energy cost of the metastable states. For example, one can directly see from Fig. 16(b) that the transition between the stripe and clusters in the equilibrium should occur at $T \approx 2.8 \mathrm{~K}$.

\section{CONCLUSIONS}

We presented novel and rich vortex phases and phase transitions in a type-I/type-II superconducting bilayer, resembling known phenomena in soft-matter physics. The solution-gel-glass-crystal transitions of vortex matter can be induced in our system by an external magnetic field, current, or temperature, but can be also engineered by a proper choice of the constituent materials, thinning the type-I layer to effective type-II behavior, or changing the spacer material to influence the coupling strength. The proposed superconducting system is in many ways peculiar and different from any soft-matter system, but similarities arise from the competing interactions with different length scales, present in both systems. Our superconducting system is controllable, relatively easy to fabricate, and allows for convenient vortex imaging or detection of transitions between phases using neutron scattering or calorimetric measurements. Moreover, this system opens a further research direction, leaning upon the early discovery of Giaver that it is possible to make a $\mathrm{dc}$ transformer by using applied current in one superconductor to drag vortices through another and induce voltage there. ${ }^{28}$ The inability to ad hoc predict what would happen to soft vortex matter phases in that case, as well as the links to related studies of Coulomb drag in semiconductor heterostructures ${ }^{29}$ and bilayer graphene, ${ }^{30}$ makes our system a very interesting test bed for a plethora of new phenomena.

\section{ACKNOWLEDGMENTS}

This work was supported by the Flemish Science Foundation (FWO-Vl). Insightful discussions with Arkady Shanenko and Edith Cristina Euan Diaz are gratefully acknowledged. *lucia.komendova@uantwerp.be

${ }^{1}$ R. A. L. Jones, Soft Condensed Matter (Oxford University Press, Oxford, 2002).

${ }^{2}$ G. Malescio and G. Pellicane, Nat. Mater. 2, 97 (2003).

${ }^{3}$ C. J. Olson Reichhardt, C. Reichhardt, and A. R. Bishop, Phys. Rev. E 82, 041502 (2010).

${ }^{4}$ H. J. Zhao, V. R. Misko, and F. M. Peeters, New J. Phys. 14, 063032 (2012); J. A. Drocco, C. J. Olson Reichhardt, C. Reichhardt, and A. R. Bishop, J. Phys.: Condens. Matter 25, 345703 (2013).

${ }^{5}$ C. N. Varney, K. A. H. Sellin, Qingze Wang, H. Fangohr, and E. Babaev, arXiv:1212.1130v1.

${ }^{6}$ P. J. Camp, Phys. Rev. E 68, 061506 (2003).

${ }^{7}$ J. C. Fernandez Toledano, F. Sciortino, and E. Zaccarelli, Soft Matter 5, 2390 (2009).

${ }^{8}$ C. L. Klix, C. P. Royall, and H. Tanaka, Phys. Rev. Lett. 104, 165702 (2010).

${ }^{9}$ N. Osterman, D. Babič, I. Poberaj, J. Dobnikar, and P. Ziherl, Phys. Rev. Lett. 99, 248301 (2007); J. Dobnikar, J. Fornleitner, and G. Kahl, J. Phys.: Condens. Matter 20, 494220 (2008).

${ }^{10}$ R. Prozorov, Phys. Rev. Lett. 98, 257001 (2007); R. Prozorov, A. F. Fidler, J. R. Hoberg, and P. C. Canfield, Nat. Phys. 4, 327 (2008); G. R. Berdiyorov, A. D. Hernandez, and F. M. Peeters, Phys. Rev. Lett. 103, 267002 (2009).

${ }^{11}$ G. Blatter, M. V. Feigel'man, V. B. Geshkenbein, A. I. Larkin, and V. M. Vinokur, Rev. Mod. Phys. 66, 1125 (1994).

${ }^{12}$ V. V. Moshchalkov, M. Menghini, T. Nishio, Q. H. Chen, A. V. Silhanek, V. H. Dao, L. F. Chibotaru, N. D. Zhigadlo, and J. Karpinski, Phys. Rev. Lett. 102, 117001 (2009); J. Gutierrez, B. Raes, A. V. Silhanek, L. J. Li, N. D. Zhigadlo, J. Karpinski, J. Tempere, and V. V. Moshchalkov, Phys. Rev. B 85, 094511 (2012); T. Nishio, Vu Hung Dao, Q. Chen, L. F. Chibotaru,
K. Kadowaki, and V. V. Moshchalkov, ibid. 81, 020506(R) (2010).

${ }^{13}$ M. Sigrist and K. Ueda, Rev. Mod. Phys. 63, 239 (1991).

${ }^{14}$ V. B. Eltsov, R. Blaauwgeers, N. B. Kopnin, M. Krusius, J. J. Ruohio, R. Schanen, and E. V. Thuneberg, Phys. Rev. Lett. 88, 065301 (2002).

${ }^{15}$ E. H. Brandt, Phys. Rev. B 79, 134526 (2009).

${ }^{16}$ A. Chaves, F. M. Peeters, G. A. Farias, and M. V. Milošević, Phys. Rev. B 83, 054516 (2011); J. Carlström, J. Garaud, and E. Babaev, ibid. 84, 134515 (2011)

${ }^{17} \mathrm{~W}$. Lawrence and S. Doniach, in Proceedings of the Twelfth International Conference on Low Temperature Physics, edited by Eizo Kanda (Academic Press of Japan, Kyoto, 1971), p. 361 .

${ }^{18}$ R. A. Klemm, A. Luther, and M. R. Beasley, Phys. Rev. B 12, 877 (1975).

${ }^{19}$ L. N. Bulaevskii and I. D. Vagner, Phys. Rev. B 43, 8694 (1991).

${ }^{20}$ S. J. Chapman, Q. Du, and M. D. Gunzburger, SIAM J. Appl. Math. 55, 156 (1995).

${ }^{21}$ M. J. Graf, D. Rainer, and J. A. Sauls, Phys. Rev. B 47, 12089 (1993).

${ }^{22}$ H. Bluhm, N. C. Koshnick, M. E. Huber, and K. A. Moler, Phys. Rev. Lett. 97, 237002 (2006).

${ }^{23}$ M. V. Milošević and R. Geurts, Physica C 470, 791 (2010).

${ }^{24}$ K. A. H. Sellin and E. Babaev, arXiv:1308.2109v1.

${ }^{25}$ M. Menghini and R. J. Wijngaarden, Phys. Rev. B 72, 172503 (2005).

${ }^{26}$ J. R. Hoberg and R. Prozorov, Phys. Rev. B 78, 104511 (2008).

${ }^{27}$ V. Vlasko-Vlasov, A. Buzdin, A. Melnikov, U. Welp, D. Rosenmann, L. Uspenskaya, V. Fratello, and W. Kwok, Phys. Rev. B 85, 064505 (2012); V. Vlasko-Vlasov, U. Welp, G. Karapetrov, V. Novosad, D. Rosenmann, M. Iavarone, A. Belkin, and W.-K. Kwok, ibid. 77, 134518 (2008). 
${ }^{28}$ Ivar Giaver, Phys. Rev. Lett. 15, 825 (1965); R. Deltour and M. Tinkham, Phys. Rev. 174, 478 (1968); J. W. Ekin, B. Serin, and J. R. Clem, Phys. Rev. B 9, 912 (1974).

${ }^{29}$ P. M. Solomon, P. J. Price, D. J. Frank, and D. C. La Tulipe, Phys. Rev. Lett. 63, 2508 (1989); T. J. Gramila, J. P. Eisenstein, A. H. MacDonald, L. N. Pfeiffer, and K. W. West, ibid. 66, 1216 (1991).

${ }^{30}$ R. V. Gorbachev, A. K. Geim, M. I. Katsnelson, K. S. Novoselov, T. Tudorovskiy, I. V. Grigorieva, A. H. MacDonald, S. V. Morozov,
K. Watanabe, T. Taniguchi, and L. A. Ponomarenko, Nat. Phys. 8, 896 (2012).

${ }^{31}$ Ben Xu, M. V. Milošević, and F. M. Peeters, Phys. Rev. B 81, 064501 (2010); Ben Xu, M. V. Milošević, Shi-Hsin Lin, F. M. Peeters, and B. Jankó, Phys. Rev. Lett. 107, 057002 (2011).

${ }^{32}$ O. Bourgeois, S. E. Skipetrov, F. Ong, and J. Chaussy, Phys. Rev. Lett. 94, 057007 (2005). 